\title{
Label-free fiber optic biosensor based on thin-core modal interferometer
}

\author{
Wenjie Yu, Tingting Lang, Jicheng Bian, Wen Kong \\ Institute of Optoelectronic Technology, China Jiliang University, Hangzhou 310018, China
}

\begin{abstract}
A label-free, cost-effective fiber optic biosensor (FOB) using electrostatic self-assembly (ESA) technology is presented and experimentally demonstrated. The FOB was constructed by sandwiching a thin-core single-mode fiber (TCSMF) between two single-mode fibers (SMFs). Firstly, we simulated the refractive index (RI) sensitivity of this fiber structure, and validated it using different concentrations of glycerol solutions. Then the diallyldimethyl ammonium chloride (PDDA) and styrenesulfonate sodium salt (PSS) were employed as polyelectrolyte self-assembled multilayers (PSAMs) deposition for surface functionalization of the fiber. We selected biotin-streptavidin as a bioconjugate pair for testing the effectiveness of the biosensor, and achieved a streptavidin detection limit of $0.02 \mathrm{nM}$. The specificity was further verified by a comparison experiment conducted using bovine serum albumin (BSA) and gelatin. These results demonstrate the feasibility of this sensor for use in biological and chemical applications.
\end{abstract}

Keywords: Thin-core single-mode fiber; Fiber optic biosensor; Polyelectrolyte self-assembled multilayers; Biotin-streptavidin; Specifity

\section{Introduction}

Due to their small size, anti-electromagnetic interference, high sensitivity and stability, fiber optic biosensors (FOBs) have been widely employed in the field of biological applications, especially in the areas of clinical diagnosis [1], environment protection [2], and food security [3]. Various methods of FOB construction have been reported, such as long period fiber gratings (LPFG) [4], tilted fiber Bragg gratings (TFBG) [5] and modal interference-based FOBs. Modal interference-based FOBs include Fabry-Perot interferometers (FPIs) [6], Michelson interferometers (MIs) [7], and Mach-Zehnder interferometers (MZIs) [8]. MZIs, in particular, exhibit numerous advantages over other types of FOBs, such as variable fiber structure, cost-effective fabrication, and high sensitivity. For example, a waist-enlarged fiber taper designed to detect immunoglobulin G [9] and a single mode tapered fiber designed to detect protein (gelatin) [10] have been realized. Unfortunately, the fabrication of these tapered structures is relatively complex. On the other hand, thin-core single-mode fiber (TCSMF) based MZIs are much easier to construct, and have been used for detection of various bio-chemical materials, such as target DNA using probe DNA immobilization [1,11]. However, details of this biosensor's performance were not discussed, including detection limit, specificity validation, and so on.

One important preparation step for FOBs is the surface functionalization of the fiber. Usually, the Langmuir-Blodgett (LB) technique [12] or the electrostatic self-assembly (ESA) technique, otherwise known as polyelectrolyte self-assembled multilayers (PSAMs) deposition [9] is utilized. While LB films possess relatively poor mechanical and thermal stability, PSAMs have strong layer-by-layer ionic bonds that enhance the film quality, making it a stronger candidate for use. ESA has been studied extensively since 1992, when Decher first successfully prepared PSAMs through the alternating electrostatic adsorption of a polycation and polyanion [13]. In addition to enhanced film quality, 
PSAMs permit a diverse array of materials, such as the displaced molecules, ions, and proteins, to be passed through them, providing an ideal platform for interaction between the fiber probe and its surrounding materials, particularly in direct protein immobilization. It has also been implemented with success in numerous other chemical applications, including in the measurement of $\mathrm{pH}$ [14], relative humidity (RH) [15], and refractive index (RI) [16].

Biotin-streptavidin system has been widely used for biological applications, and many detection approaches with FOBs exists [4, 5, 17]. For example, the turnaround point (TAP) LPFG was fabricated [4]. It can improve the detection sensitivity, but the complicated manufacturing procedure is a problem. Other biosensors were made with TFBG coatingwith gold nanoparticles [5] or LPFG anchored with silica core gold shell nanoparticles [17]. However, despite the sensors' effectiveness, the high fabrication costs required for sensor production have limited further development.

In this paper, a simple fiber optical biosensor based on a thin-core single-mode fiber sandwiched between two single-mode fibers (SMF-TCSMF-SMF) was proposed. The RI sensitivity of the fiber sensor was first validated through simulation and experiment. Then the TCSMF was modified with PSAMs, and then modified with biotin, which functioned as a ligand for streptavidin detection. Streptavidin concentrations were detected by measuring the refractive index changes caused by the binding of streptavidin to biotin through experiment. The bio-information of this interaction was detected by monitoring shifts in resonance wavelength. The specificity of this fiber optical biosensor was also verified experimentally.

\section{Materials and Methods}

\subsection{Sensor principle and RI sensitivity validation}

Fig. 1 provides a schematic illustration of the proposed MZI-based fiber optic sensor: a TCSMF with the length $L$ sandwiched between two SMFs, all ends are not tapered. Because the cladding diameter of the TCSMF is the same as that of the SMFs $(125 \mu \mathrm{m})$, we can construct the fiber probe using a commercial fusion splicer in edge-aligned mode, making fabrication simple and effective. The core diameters of the SMFs and TCSMF are $8.2 \mu \mathrm{m}$ and $2.1 \mu \mathrm{m}$, respectively. The TCSMF has a length of $20 \mathrm{~mm}$.

This is one of the most common MZI configurations because the two interfaces function as mode splitter and combiner, respectively. The different core diameters allow some of the input light to leak into the TCSMF's cladding at the first interface, thus stimulating the cladding mode. After transferring along the distance $L$, light propagating in the cladding is reconstituted with the core mode at the second interface, generating an intermodal interference signal due to the optical path difference. If the sensor is connected to an optical spectrum analyzer for signal processing, the transmission spectrum can be observed, its peaks and dips corresponding to the constructive and destructive interference phases.

In analysis of the transmission spectrum, the interferometric phase matching condition can be defined as

$$
2 \pi\left[n_{e f f}^{c o}(\lambda)-n_{\text {eff }}^{c l, j}\left(\lambda, n_{\text {ext }}\right)\right] \frac{L}{\lambda_{D}}=(2 k+1) \pi
$$

Where $L$ is the length of the TCSMF, $n_{e x t}$ is the refractive index of the surrounding medium, $\lambda_{D}$ is the interferometric wavelength of transmission, $k$ is an integer, and $n_{\text {eff }}^{c o}(\lambda)$ and $n_{\text {eff }}^{c l, j}\left(\lambda, n_{\text {ext }}\right)$ are the effective refractive indices of the core mode and the $j$ th order cladding mode, respectively. It is well 
known that $n_{\text {eff }}^{c o}(\lambda)$ is not sensitive to the external refractive index (ERI), but $n_{\text {eff }}^{c l, j}\left(\lambda, n_{\text {ext }}\right)$ is. This is what enables us to utilize this structure for refractive index sensing, even in biological testing. The sensitivity of the transmission dip to changes in the surrounding refractive index can be calculated using Eq. (1)

$$
S=\frac{d \lambda}{d n_{e x t}}=\frac{\frac{-\lambda_{D}}{\Delta n_{e f f}} \frac{\partial n_{e f f}^{c l, j}}{\partial n_{e x t}}}{\left[1-\frac{\lambda_{D}}{\Delta n_{e f f}}\left(\frac{\partial n_{e f f}^{c o}}{\partial \lambda}-\frac{\partial n_{e f f}^{c l, j}}{\partial \lambda}\right)\right]}
$$

Where $\Delta n_{\text {eff }}$ is the difference in the effective indices between the core and the $j$ th order cladding mode. As the value of $n_{e x t}$ increases, the value of $\Delta n_{e f f}$ decreases, resulting in the enhancement of $S$ according to Eq. (2). In other words, in theory, there is no linear relationship between interferometric wavelength and ERI. This conclusion is also verified through the next simulation and experiment described.

The fiber probe's simulation is based on the modal propagation analysis method [18]. And the parameters used in the simulation are listed in Table 1.

\begin{tabular}{ccc}
\hline & SMF & TCSMF \\
\hline Length $(\mathrm{mm})$ & 0.5 & 20 \\
\hline RI of the core & 1.4512 & 1.4504 \\
\hline RI of the cladding & 1.4447 & 1.4447 \\
\hline
\end{tabular}

Table 1. Parameters of the fiber optic sensor used in simulation

Assuming the light in the input SMF has a fundamental mode field distribution $E(r, 0)$ and the field profile within TCSMF is $\Psi(r)$, the input field at the length of TCSMF $z$ can be written as

$$
\begin{aligned}
\psi(\mathrm{r}) & =\sum_{j=1}^{M} \psi_{j}(r) \\
E(\mathrm{r}, z) & =\sum_{j=1}^{M} b_{j} \psi_{j}(r) \exp \left(i \beta_{\mathrm{j}} z\right)
\end{aligned}
$$

Where $b_{j}$ is the excitation coefficient for each mode, $\Psi_{j}(r)$ is the $j$ th eigenmode of TCSMF, $\beta_{j}$ is the propagation of each eigenmode within the TCSMF. The transmitted power to the output SMF thus can be expressed as

$$
P_{\text {out }}(z)=\frac{\left|\int_{0}^{\infty} E(r, z) E(r, 0) r d r\right|^{2}}{\int_{0}^{\infty}|E(r, z)|^{2} r d r \int_{0}^{\infty}|E(r, 0)|^{2} r d r}
$$

The change of the ERI leads to the variation of the cladding mode propagation constant and influences the excitation coefficient, so output interference spectrum shifts according to Eq. (4) and (5). As shown in Fig.2 (a), with the increasement of the ERI, the transmission spectrum red shifts. In 
particular, from the relationship between the dip wavelength and ERI in Fig.2 (b), we can clearly see that the rate of the increase at lower ERI is less than that at higher ERI, which is the same as the conclusion of Eq. (2). The minimum and maximum RI sensitivities are $60 \mathrm{~nm} / \mathrm{RIU}$ at the ERI of 1.3300 and $999 \mathrm{~nm} / \mathrm{RIU}$ at the ERI of 1.4200 .

The sensor probe used in the experiment, including ordinary SMF (Corning SMF-28) and the TCSMF (Nufern 405-HP) with core diameter of $2.1 \mu \mathrm{m}$ and cutoff wavelength of $370 \pm 20 \mathrm{~nm}$. First, to make sure the sandwiched structure could be conveniently connected to a commercial fusion splicer using the edge-alignment mode (Fujikura, FSM-60S), the ends of each fiber were cleanly sliced using a knife (FC-6S). The length of TCSMF required was $20 \mathrm{~mm}$. The fiber probe was then strained using clamps and placed on a translation stage. One end of the probe was connected to a broadband light source with a wavelength range of $650 \mathrm{~nm}$ to $1650 \mathrm{~nm}$ (BBS, HOYATEK) and the other to an optical spectrum analyzer (OSA, YOKOGAWA AQ6370C) with a wavelength resolution of $0.02 \mathrm{~nm}$. A glass slide was also placed on the stage to serve as a substrate, carrying different glycerol solutions into the middle of the probe so that the TCSMF could be immersed. For calibration of the RI sensitivity characteristics of the fiber structure, the refractive indices of the glycerol solutions were measured with an Abbe refractometer (2WAJ) before.

Fig. 3 illustrates the relationship between transmission dip wavelengths and glycerol refractive indices ranging from 1.3300 to 1.3800 . The error bars were calculated by standard deviation through three similar experiments. The experimental results show that the greater the refractive index, the higher the sensitivity, verifying the accuracy of the Eq. (2). The minimum and maximum RI sensitivities in this range are $61 \mathrm{~nm} / \mathrm{RIU}$ at the ERI of 1.3300 and $545 \mathrm{~nm} / \mathrm{RIU}$ at the ERI of 1.3800 . The variation trend of the RI sensitivity is consistent with the simulation results.

\subsection{Biosensor fabrication}

Diallyldimethyl ammonium chloride (PDDA), styrenesulfonate sodium salt (PSS), biotin, and streptavidin (SA) were purchased from Sigma-Aldich (Shanghai, China). Glycerol, ethanol, 30\% hydrogen peroxide $\left(\mathrm{H}_{2} \mathrm{O}_{2}\right), 98 \%$ concentrated sulfuric acid $\left(\mathrm{H}_{2} \mathrm{SO}_{4}\right)$ and phosphate buffer saline (PBS) were purchased from Jiachen Chemical Industry, Inc. (Hangzhou, China). Bovine serum albumin (BSA) and gelatin were purchased from Dingguo Changsheng Biotechnology, Inc. (Beijing, China). All samples were prepared using either PBS or deionized (DI) water obtained from a Milli-Q water purification system.

The PSAMs used in this study consist of the polycation PDDA and the polyanion PSS, defined as $(\mathrm{PDDA}+\mathrm{PSS})_{\mathrm{n}}$, where $n$ is the number of bilayers. At $\sim 10 \mathrm{~nm}$, (PDDA+PSS) film [19] is relatively thicker than other ionic bilayers such as (PAH+PCBS) ${ }_{1}$, which is $\sim 1.5 \mathrm{~nm} \mathrm{[4]}$, and (PAH+PSS) ${ }_{1}$, which is $\sim 3.3 \mathrm{~nm}$ [20]. Therefore, it is possible to achieve the desired number of bilayers and save time by running fewer cycles.

The PSAM deposition process is shown in Fig. 4. The fiber probe was first pretreated with piranha solution (a mixture of $98 \% \mathrm{H}_{2} \mathrm{SO}_{4}$ and $30 \% \mathrm{H}_{2} \mathrm{O}_{2}$ in a $7: 3$ bulk ratio) for $1 \mathrm{~h}$, developing surface negatrons as a consequence. Then, after being washed in DI water and dried naturally, it was immersed in a solution of PDDA ( $2 \mathrm{mg} / \mathrm{ml}$ in $0.5 \mathrm{M} \mathrm{NaCl})$ for $8 \mathrm{~min}$. Any displaced molecules were removed through washing with DI water for $1 \mathrm{~min}$. Then it was immersed in PSS solution $(2 \mathrm{mg} / \mathrm{ml}$ in $0.5 \mathrm{M} \mathrm{NaCl}$ ) for $8 \mathrm{~min}$, again washed with DI water, thus completing one bilayer of PSAM film. This process was repeated until the required number of bilayers was obtained. 
Fig. 5 shows the results of the bare fiber treated with piranha solution and with (PDDA+PSS) 2 It can be seen that after removal from the piranha solution, the wavelength shifts along the short direction, but shifts in the opposite direction after the fiber was coated with PSAMs because of the ERI increasment.

To validate that our design was capable of performing as a biosensor, we choose biotin-streptavidin as a bioconjugate pair for demonstration. Accompanied by (PDDA+PSS) $)_{2}$, the sensing probe was immersed for $1 \mathrm{~h}$ in $1 \mathrm{mg} / \mathrm{ml}$ biotin solution prepared in PBS to achieve bio-functionality. Because the isoelectric point of biotin is 3.5 [5], which is relatively lower than that of PBS ( 7.5), the biotin is weakly charged and absorbed onto the PSAMs through electrostatic interaction. Subsequently, the PSAMs were rinsed with PBS to remove any unbound molecular chains of biotin. All experiments were carried out at room temperature. Moreover, it has been experimentally confirmed that the SMF-TCSMF-SMF construction is insensitive to the temperature [21], and is suitable for biological measurements.

\section{Results and discussion}

\subsection{Biotin-streptavidin binding}

To analysis definite binding capability of biotin-streptavidin in SMF-TCSMF-SMF system, several concentrations of streptavidin solutions $(1.67 \mathrm{nM} 83.3 \mathrm{nM})$ were prepared. The immersion time for each concentration was controlled at $30 \mathrm{~min}$, following by the PBS wash of 1 min to remove any unbound streptavidin. The insets of Fig. 6 (a) and (b) present the transmission spectra from 1490 $\mathrm{nm}$ to $1530 \mathrm{~nm}$. There are two resonant wavelength dips on account of Eq. (1) that different $k$ will generate various $\lambda_{D}$. Their extinction ratios are both more than $7 \mathrm{~dB}$. A clear wavelength shift is observed, which confirms the effectiveness of our proposed FOB. The enlarged spectrum of Dip A and Dip B are also shown, and we can see that the higher the concentration of streptavidin, the longer the dip wavelength, which is consistent with above simulation and glycerol experiment. The reasons of changes of intensity in spectrum response can be explained from defective fiber structure compared with simulation model and influences of other physical absorption [8].

The binding affinity and cooperative effects can be obtained using Hill model [22]. The Hill equation is described as follows

$$
\Delta \lambda=\Delta \lambda_{\max } \cdot \frac{[S A]^{n}}{\left\{K_{D}+[S A]^{n}\right\}}
$$

Where the maximum dip wavelength shift $\Delta \lambda_{\max }$ is the saturation value; $[S A]$ is the concentration of streptavidin; $n$ is the Hill coefficient; $K_{D}$ is the dissociation constant.

From the curve fitting in Fig.7, we calculate the $n$ of 0.69 and 0.84 for Dip A and Dip B, respectively. These characterize the binding between biotin and streptavidin as negative cooperativity $(n<1)$, which suggest that more than one immobilized biotins are constructed to bind one streptavidin molecule. This brings into correspondence with actual binding mechanism that $\sim 4$ biotin molecules bound to one streptavidin molecule [23]. Considering Dip A, the dissociation constant $K_{D}$ we calculate from the fitting is $3.26 \mathrm{nM}$, which is the same order of magnitude as dual-ring sensor's [22]. What's more, $\Delta \lambda_{\max }$ is about $3.39 \mathrm{~nm}$, which indicates the maximum dip wavelength shifts at the saturation concentration. Similar results have been obtained for Dip B, with the $K_{D}$ of $2.62 \mathrm{nM}$ and $\Delta \lambda_{\max }$ of 3.47 
$\mathrm{nm}$. Those resonant wavelength dips' Hill fitting results are consistent that confirm this experiment's reliability and accuracy.

\subsection{Sensitivity and detection limit}

The sensor's sensitivity, $S$, can be deduced as follows [9]

$$
S=\frac{\Delta \lambda_{\max }}{\delta_{\max }}
$$

Where $\Delta \lambda_{\max }$ is $3.39 \mathrm{~nm}$, the maximum dip wavelength shift at Dip A; $\delta_{\max }$ is the surface density of the streptavidin when all binding sites of immobilized biotin are occupied [24]. It can be determined by [9]

$$
\delta_{\text {max }}=\frac{M_{S A}}{N_{A} \cdot P_{S A}^{2}}
$$

Where $M_{S A}=60000 \mathrm{~g} / \mathrm{mol}$ is the estimated molecular mass of the streptavidin [25], $N_{A}=6.02 \times 10^{23}$ $\mathrm{mol}^{-1}$ is the Avogadro's number and $P_{S A}=4 \mathrm{~nm} \mathrm{[26]} \mathrm{is} \mathrm{the} \mathrm{average} \mathrm{length} \mathrm{of} \mathrm{one} \mathrm{streptavidin} \mathrm{molecule,}$ hence the $\delta_{\max }$ is calculated to be $6.23 \mathrm{ng} / \mathrm{mm}^{2}$ and the streptavidin detection sensitivity for SMF-TCSMF-SMF structure is calculated to be $0.55 \mathrm{~nm} /\left(\mathrm{ng} / \mathrm{mm}^{2}\right)$.

The $K_{D}$ obtained with Hill model show that dissociation constant is strongly related to the biotin-streptavidin recognition; also to the streptavidin detection limit $C_{\text {lim }}$ that is [24]

$$
C_{\lim }=K_{D} \cdot \frac{S_{\lambda}}{\Delta \lambda_{\text {max }}-S_{\lambda}}
$$

Where $S_{\lambda}$ is the OSA resolution of $0.02 \mathrm{~nm}$. It can be seen from Eq. (9) that smaller dissociation constant results in lower detection limit. Thus $C_{\text {lim }}$ is calculated to be $0.02 \mathrm{nM}$ according to the conclusions of Dip A, which is lower than the $19 \mathrm{nM}$ limit of the LFPG biosensor [17].

For improving the sensitivity and detection limit, more studies will be deepened in the future. It has been reported that the RI sensitivity could be improved 7.1 times better than our structure's, if the TCSMF's cladding was etched by hydrofluoric acid (HF) solution [27]. We regard this as the next research step to apply to biosensing.

\subsection{Specificity validation}

To further confirm the specificity, we selected gelatin and bovine serum albumin (BSA) as comparative proteins for experiment respectively. Fig. 8 (a) is the changes of dip wavelength in each above mentioned experimental step following each immersion time. The concentrations of the three protein solutions (gelatin, BSA and SA) are all $5 \mathrm{nM}$. Clearly, after the fiber is functionalized with biotin, the transmission spectrum shift of the SA is much longer than that of gelatin and BSA. The dip wavelength shifts for the three solutions are shown in Fig. 8 (b), which is about $0.04 \mathrm{~nm}, 0.08 \mathrm{~nm}$ and $1.48 \mathrm{~nm}$ respectively. The resonant wavelength shifts were not obvious after immersing the fiber biosensor in BSA and gelatin. The dip wavelength shift of SA is about 37 and 18 times bigger than that of gelatin and BSA, which indicates the high specificity of our proposed fiber optical biosensor. In addition, Fig. 8 shows the possibility of this biosensor to be applied in real-time detection.

\section{Conclusion}

Using ESA technology, a SMF-TCSMF-SMF structure coated with PSAMs was built, producing a 
streptavidin biosensor with detection limit as low as $0.02 \mathrm{nM}$. The fiber probe was functionalized using (PDDA+PSS $)_{2}$, possessing a number of advantages - cost-effectiveness, ease of fabrication, high sensitivity, specificity, label-free, and so on. The proposed sensor provides an attractive platform for biosensing and may also be used for detection in chemical and biological fields.

\section{Acknowledgements}

This work was supported by the National Science Foundations of China under grant 61205029 .

\section{References}

[1] W. K. EdwardáChan, A. PingáZhang, Label-free, disposable fiber-optic biosensors for DNA hybridization detection, Analyst 138 (2013) 1988-1994.

[2] S. K. Mishra, S. Rani, B. D. Gupta, Surface plasmon resonance based fiber optic hydrogen sulphide gas sensor utilizing nickel oxide doped ITO thin film, Sensors and Actuators B: Chemical 195 (2014) 215-222.

[3] X. J. Hao, X. H. Zhou, Y. Zhang, L. H. Liu, F. Long, L. Song, H. C. Shi, Melamine detection in dairy products by using a reusable evanescent wave fiber-optic biosensor, Sensors and Actuators B: Chemical 204 (2014) 682-687.

[4] Z. Y. Wang, J. R. Heflin, K. V. Cott, R. H. Stolen, S.Ramachandran, S. Ghalmi, Biosensors employing ionic self-assembled multilayers adsorbed on long-periodfiber gratings, Sensors and Actuators B: Chemical 139 (2009) 618-623.

[5] S. Lepinay, A. Staff, A. Ianoul, J. Albert, Improved detection limits of protein optical fiber biosensors coated with gold nanoparticles, Biosensors and Bioelectronics 52 (2014) 337-344.

[6] Y. Zhang, H. Shibru, K. L. Cooper, A. Wang, Miniature fiber-optic multicavity Fabry-Perot interferomrtric biosensor, Optics Letters 30 (2005) 1021-1023.

[7] D. W. Kim, Y. Zhang, K. L. Cooper, A. Wang, Fibre-optic interferometric immuno-sensor using long period grating, Electronics letters 42 (2006) 324-325.

[8] Y. Tian, W. Wang, N. Wu, X. Zou, X. Wang, Tapered optical fiber sensor for label-free detection of biomolecules, Sensors (Basel) 11(2011) 3780-3790.

[9] L. H. Chen, C.C. Chan, K. Ni, P. B. Hu, T. Li, W. C. Wong, P. Balamurali, R. Menon, M. Shaillender, B. Neu, C. L. Poh, X. Y. Dong, X. M. Ang, P. Zu, Z. Q. Tou, K. C. Leong, Label-free fiber-optic interferometric immunosensors based on waist-enlarged fusion taper, Sensors and Actuators B: Chemical 178 (2013) 176-184.

[10] T. K. Yadav, R. Narayanaswamy, M. H. A. Bakar, Y. M. Kamil, M. A. Mahdi, Single mode tapered fiber-optic interferometer based refractive index sensor and its application to protein sensing, Optics Express 22 (2014) 22802-22807.

[11] G. Y. Ma, P. Zhang, G. F. Yan, Y. B. Bai, Y. C. Hu, X. S. Ye, Label-free DNA biosensor based on cladding-etched thin-core fiber modal interferometer, Proc. SPIE 84215(2012) 1-4 .

[12] D. R. Nicholas, W. J. Stephen, P. T. Ralph, Optical fiber long period gratings with Langmuir-Blodgett thin film overlays, Optics Letters 27 (2002) 686-688.

[13] G. Decher, Build up of ultrathin multilayer films by a self-assembly process: III. Consecutively alternating adsorption of anionic and cationic polyelectrolytes on charged surfaces, Thin Solid Films 210-211(1992) 831-835.

[14] B. B. Gu, M. J. Yin, A. P. Zhang, J. W. Qian, S. L. He, Low-cost high-performance fiber-optic 
$\mathrm{pH}$ sensor based on thin-core fiber modal interferometer, Optics Express 17 (2009) 22296-22302.

[15] S. Akita, H. Sasaki, K. Watanabe, A. Seki, A humidity sensor based on a hetero-core optical fiber, Sensors and Actuators B: Chemical 147 (2010) 385-391.

[16] Q. S. Li, X. L. Zhang, H. He, Q. J. Meng, J. G. Shi, J. N. Wang, W. F. Dong, Improved detecting sensitivity of long period fiber gratings by polyelectrolyte multilayers: The effect of film structures, Optics Communications 331 (2014) 39-44.

[17] L. Marques, F. U. Hernandez, S. Korposh, M. Clark, S. Morgan, S. W. James, R. P. Tatam, Sensitive protein detection using an optical fibre long period grating sensor anchored with silica core gold shell nanoparticles, Proc. SPIE 9157 (2014) 1-4.

[18] Q. Wu, Y. Semenova, P. F. Wang, G. Farrell, A comprehensive analysis verified byexperiment of a refractometer based on anSMF28-small-core singlemode fiber(SCSMF)-SMF28 fiber structure, Journal of Optics 13 (2011) 937-946.

[19] C. Y. Gao, S. Leporatt, S. Moya, E. Donath, H. Mohwald, Swelling and shrinking of polyelectrolyte microcapsules in response to Swelling changes in temperature and ionic strength, Chemistry 9 (2003) 915-920.

[20] W. F. Dong, J. K. Ferri, T. Adalsteinsson, M. Schonhoff, G. B. Sukhorukov, H. Mohwald, Influence of Shell Structure on Stability, Integrity, and Mesh Size of Polyelectrolyte Capsules: Mechanism and Strategy for Improved Preparation, Chem. Mater. 10 (2005) 2603-2611.

[21] B. Xu, J. Q. Li, Y. Li, J. L. Xie, X. Y. Dong, Liquid seal for temperature sensing with fiber-optic refractometer, Sensors 14 (2014) 14873-14884.

[22] K. W. Kim, J. F. Song, J. S. Kee, Q. Liu, G. Q. Lo, M. K. Park, Label-free biosensor based on an electrical tracing-assisted silicon microring resonator with a low-cost broadband source, Biosensors and Bioelectronics 46 (2013) 15-21.

[23] C. S. Neish, L. L. Martin, R. M. Henderson, and J. M. Edwardson, Direct visualization of ligand-protein interactions using atomic force microscopy, Br. J. Pharmacol 135 (2002) 1943-1950.

[24] S. Maguis, G. Laffont, P. Ferdinand, B. Carbonnier, K. Kham, T. Mekhalif, M. C.Millot, Biofunctionalized tilted Fiber Bragg Gratings for label-free immunosensing, Optics Express 16 (2008) 19049-19062.

[25] J. R. Smith, K. R. Amaya, R. T. Bredemeier, S. Banta, and D. M. Cropek, Selective biomolecular photocatalytic decomposition using peptide-modified $\mathrm{TiO} 2$ nanoparticles, Applied Catalysis B Environmental 176 (2015) 315-324.

[26] H. Y. Li, S. H. Park, J. H. Reif, T. H. Labean, and H. Yan, DNA-Templated Self-Assembly of Protein and Nanoparticle Linear Arrays, Journal of the American Chemical Society 126 (2004) 418-419.

[27] B. Xu, Y. Li, X. Y. Dong, S. Z. Jin, Z. X. Zhang, Highly Sensitive Refractive Index Sensor Based on a Cladding-Etched Thin-Core Fiber Sandwiched between Two Single-Mode Fibers, Chinese Physics Letter 29 (2012) 94203-94205.

Fig.1. Schematic diagram of the fiber optic sensor with a TCSMF sandwiched between two SMFs Fig. 2. (a) Simulated transmission spectrum versus different ERIs (b) Relationship between dip wavelength and ERI 
Fig. 3. Experimental relationship between dip wavelength and ERI

Fig. 4. PSAMs deposition process

Fig. 5. Spectra recorded of the bare fiber, after treatment in piranha solution and PAMs successively Fig. 6. Transmission spectra versus different concentrations of SA at Dip A (a) and Dip B (b). The insets are the whole spectrum from $1490 \mathrm{~nm}$ to $1530 \mathrm{~nm}$.

Fig.7 A Hill fitting based on above SA experiment.

Fig. 8. (a) Wavelength response for each experimental step versus time.

Fig. 8. (b) Dip wavelength shift versus different proteins (gelatin, BSA and SA). 


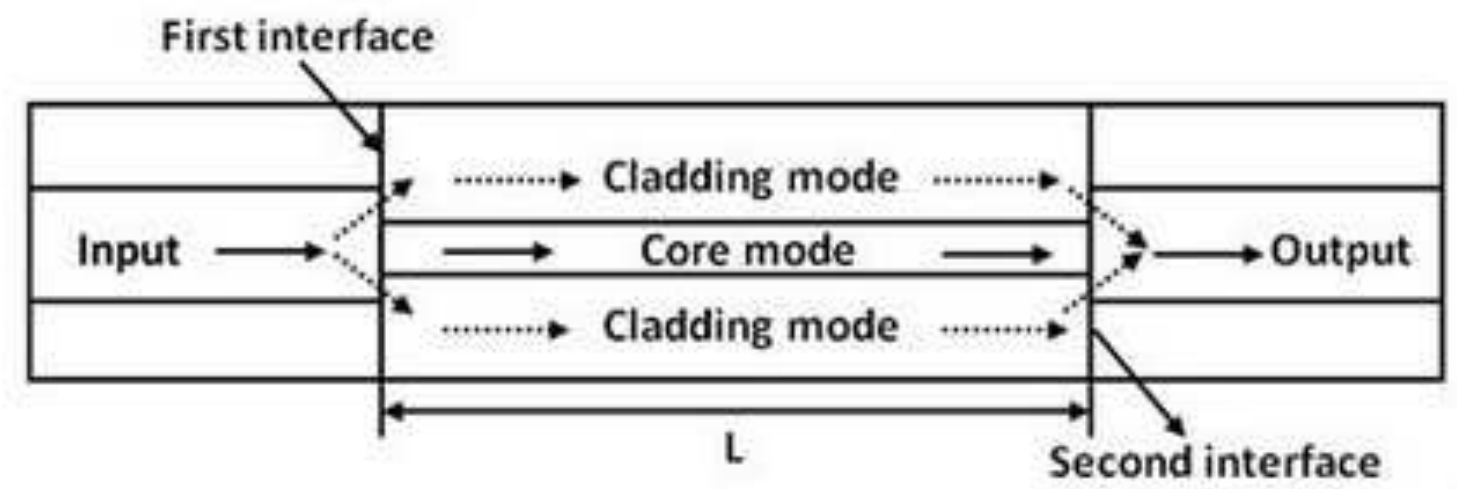

Fig.1. 


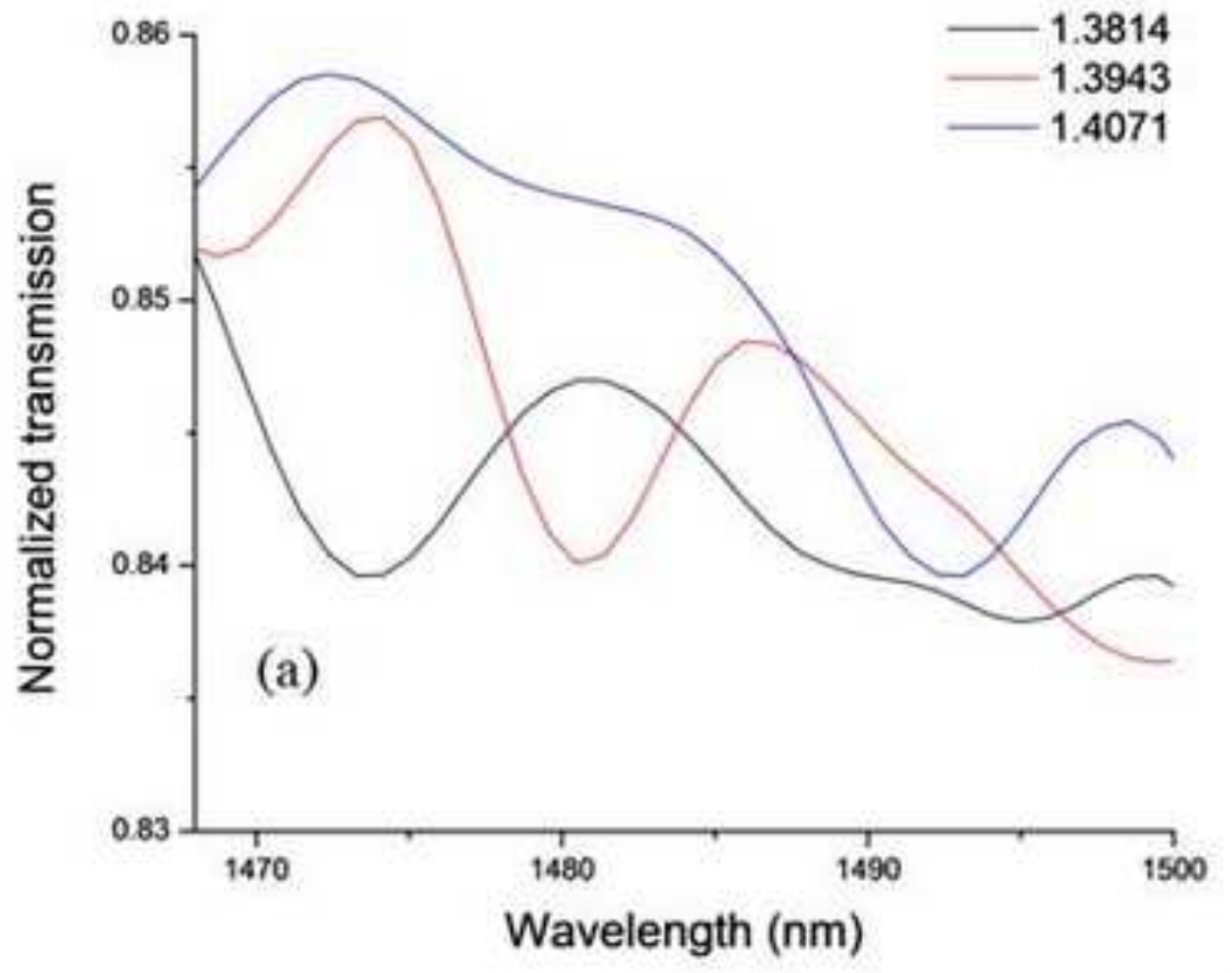

Fig. 2. (a) 


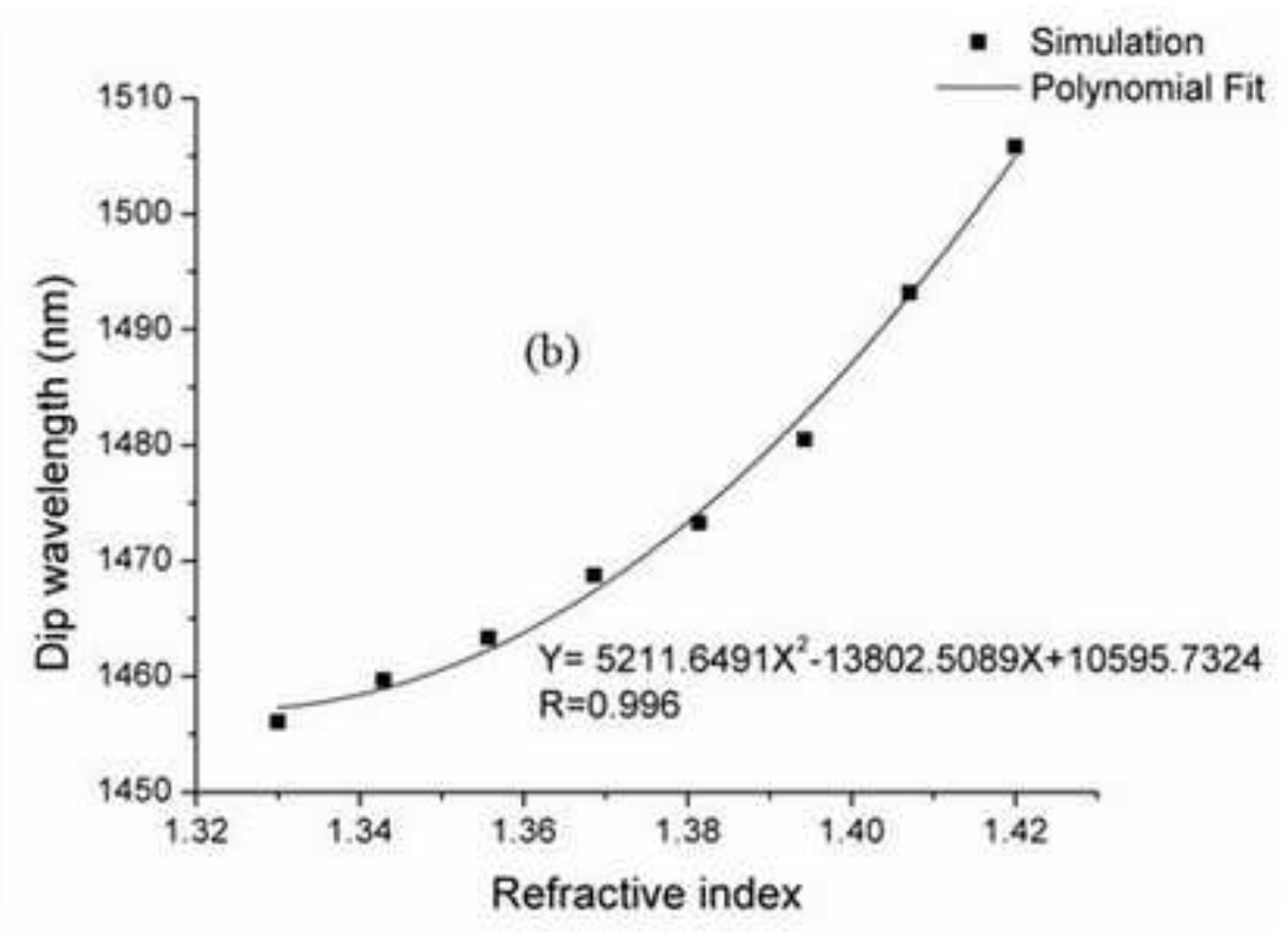

Fig. 2. (b) 


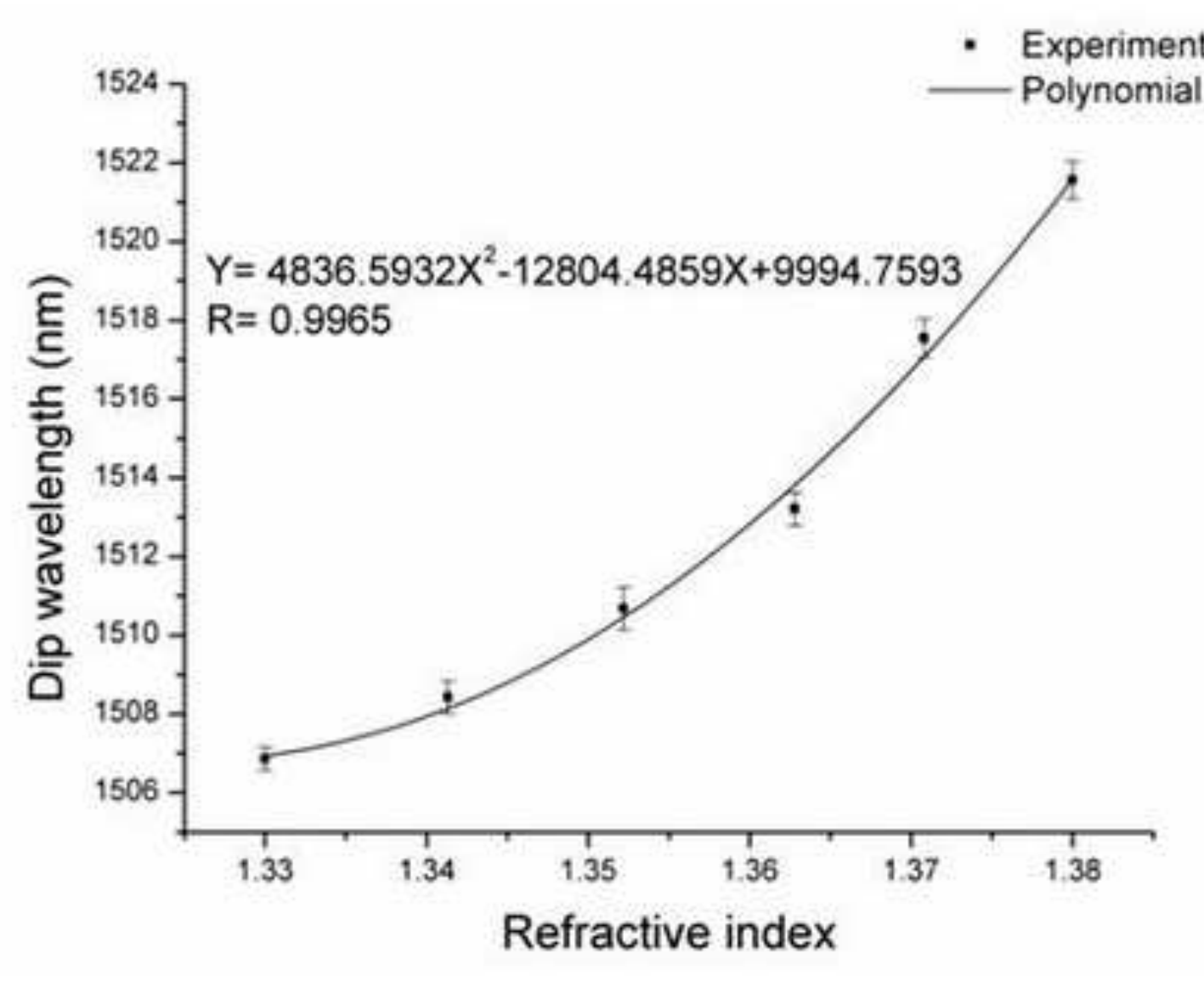

Fig. 3. 


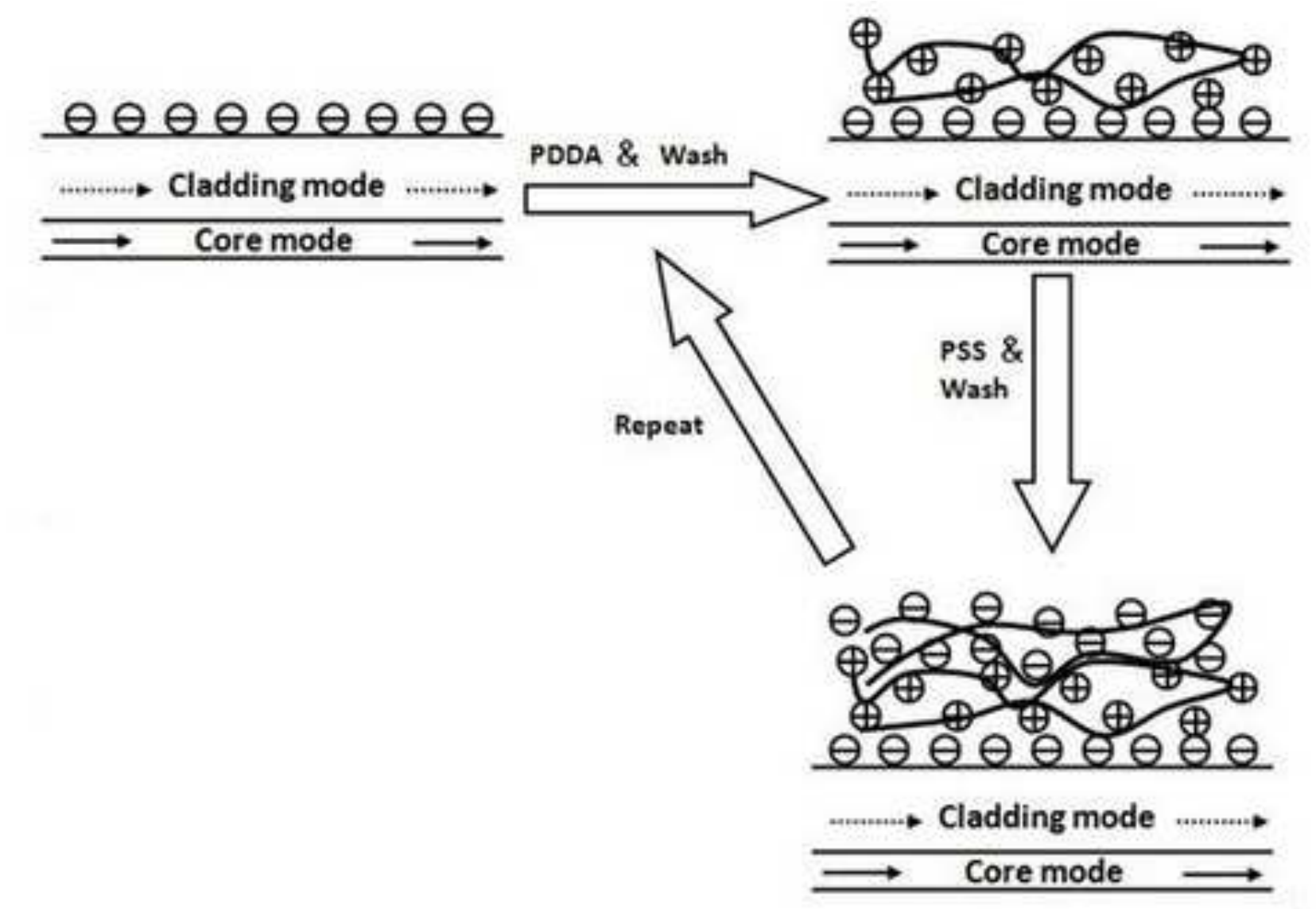

Fig. 4. 


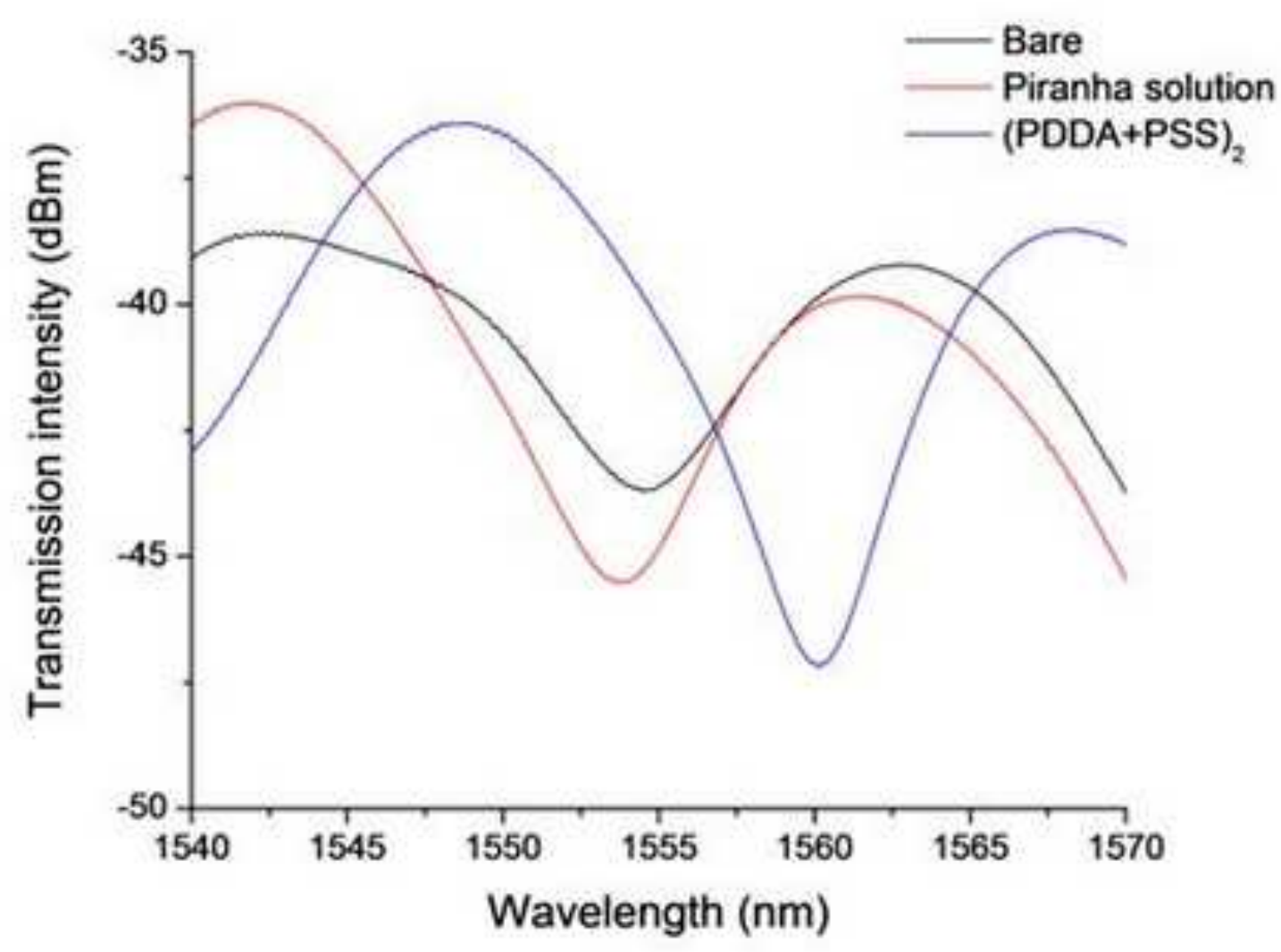

Fig. 5. 


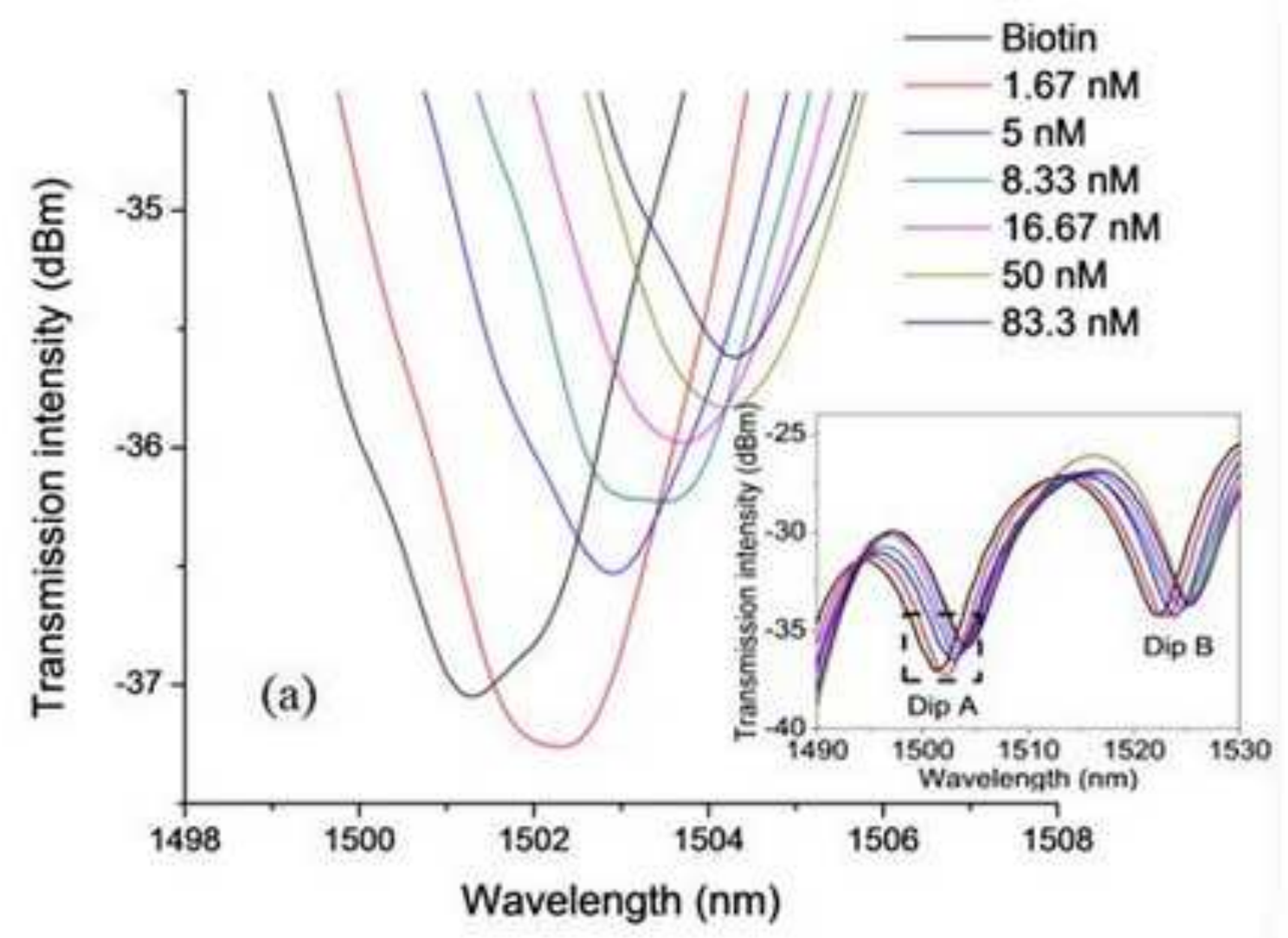

Fig. 6. (a) 


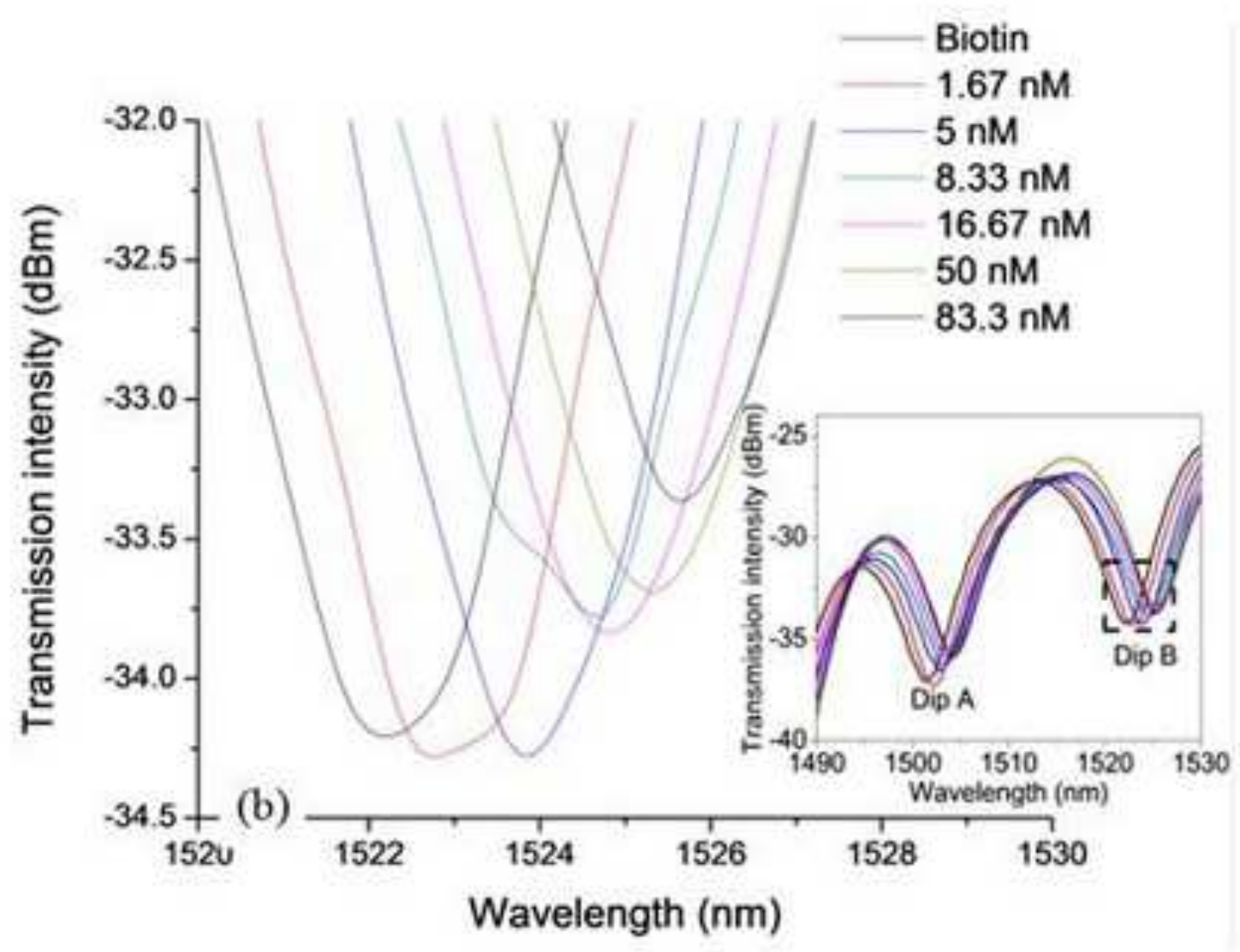

Fig. 6. (b) 


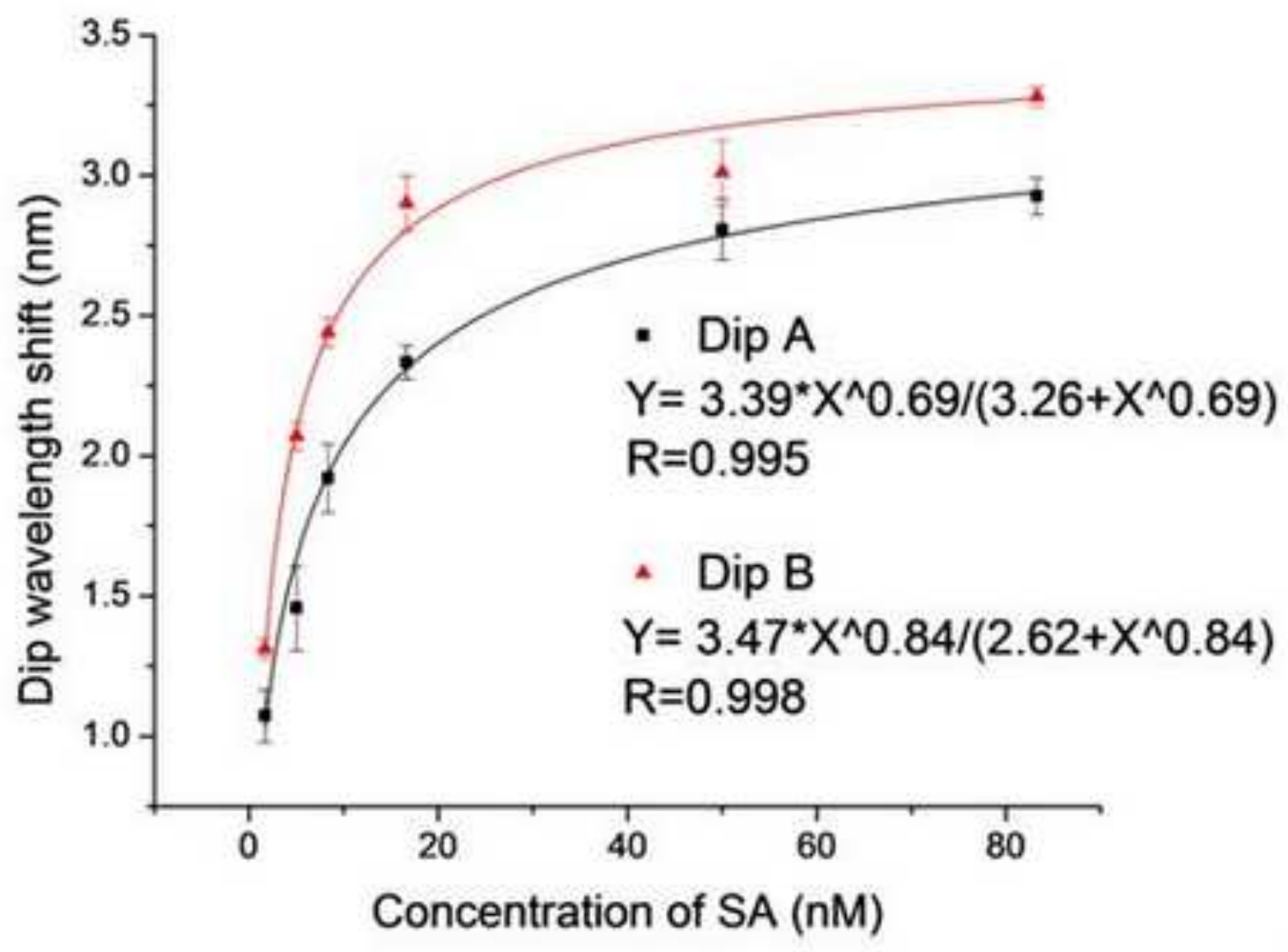

Fig.7. 


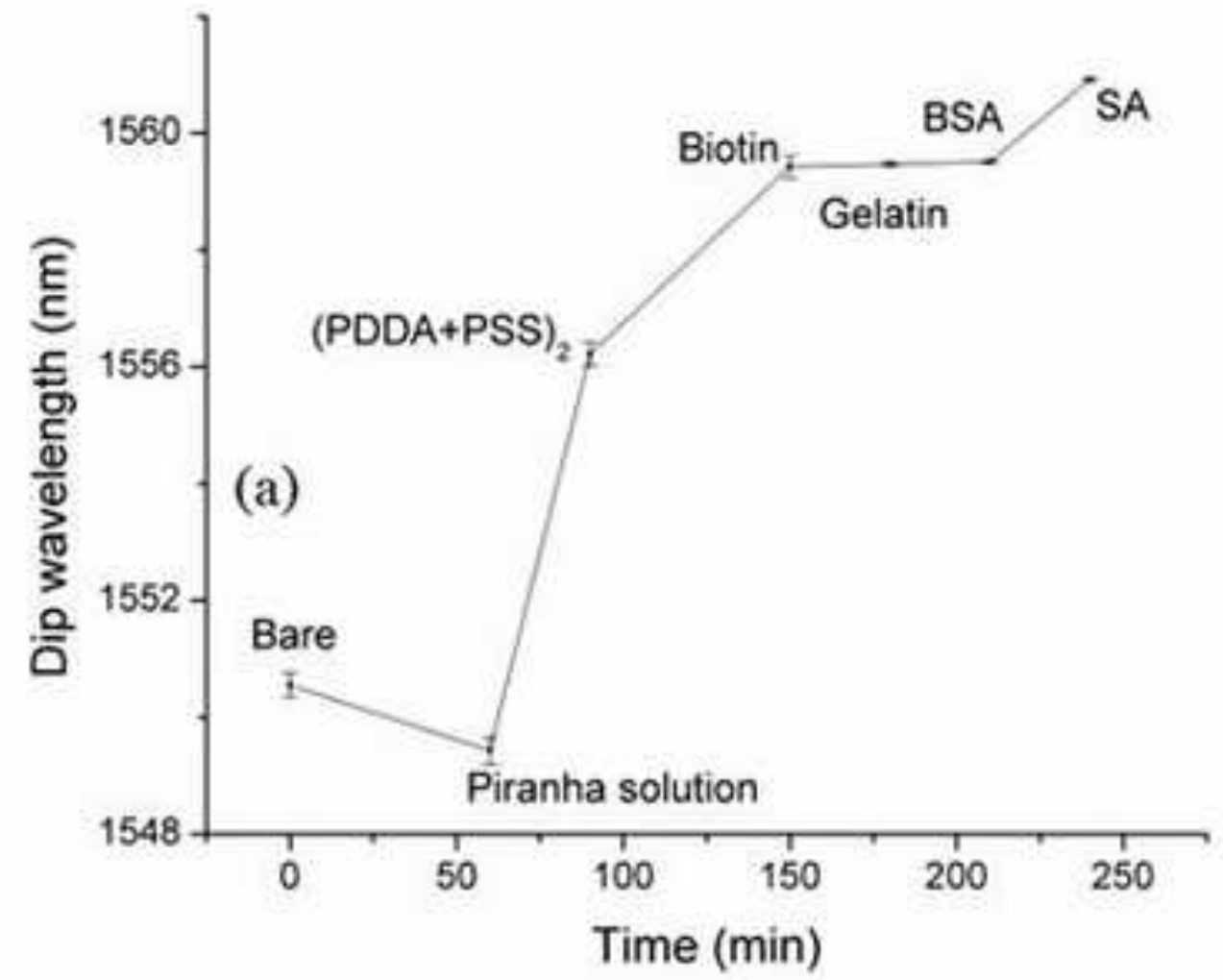

Fig. 8. (a) 


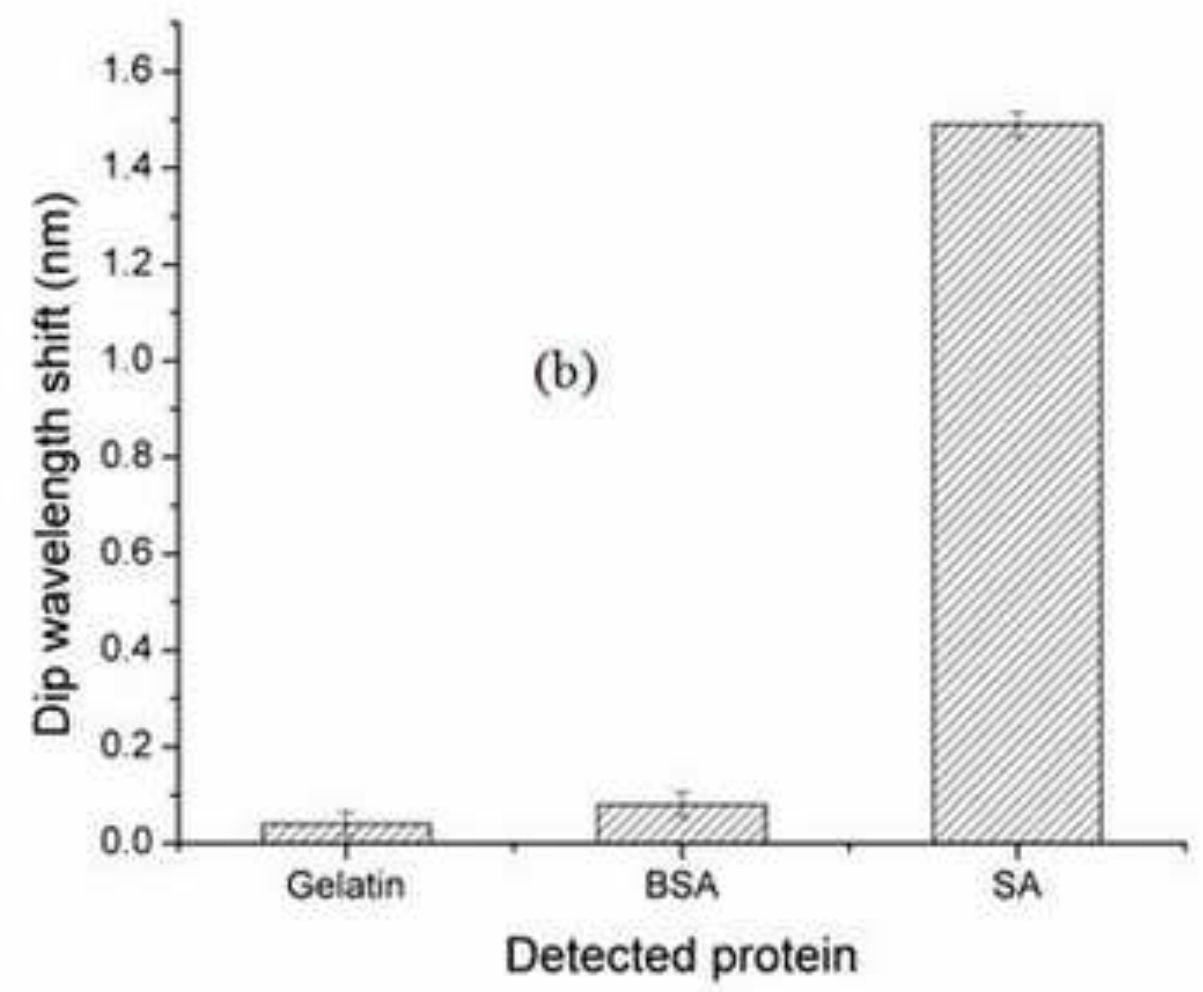

Fig. 8. (b) 